\title{
Simultaneous determination of phagocytosis of Plasmodium falciparum-parasitized and non-parasitized red blood cells by flow cytometry
}

\author{
Valentina Gallo, Oleksii A Skorokhod, Evelin Schwarzer and Paolo Arese*
}

\begin{abstract}
Background: Severe falciparum malaria anaemia (SMA) is a frequent cause of mortality in children and pregnant women. The most important determinant of SMA appears to be the loss of non-parasitized red blood cells (np-RBCs) in excess of loss of parasitized (p-) RBCs at schizogony. Based on data from acute SMA where excretion of haemoglobin in urine and increased plasma haemoglobin represented respectively less than $1 \%$ and $0.5 \%$ of total $\mathrm{Hb}$ loss, phagocytosis appears to be the predominant mechanism of removal of $\mathrm{np}$ - and $\mathrm{p}-\mathrm{RBC}$.

Estimates indicate that np-RBCs are cleared in approximately 10-fold excess compared to p-RBCs. An even larger removal of np-RBCs has been described in vivax malaria anaemia. Estimates were based on two single studies both performed on neurosyphilitic patients who underwent malaria therapy. As the share of $n p-R B C$ removal is likely to vary between wide limits, it is important to assess the contribution of both $\mathrm{np}$ - and $\mathrm{p}$-RBC populations to overall RBC loss, and disclose the mechanism of such variability. As available methods do not discriminate between the removal of np- vs p-RBCs, the purpose of this study was to set up a system allowing the simultaneous determination of phagocytosis of $\mathrm{p}$ - and $\mathrm{np}-\mathrm{RBC}$ in the same sample.

Methods and Results: Phagocytosis of $\mathrm{p}$ - and np-RBCs was quantified in the same sample using double-labelled target cells and the human phagocytic cell-line THP-1, pre-activated by TNF and IFNy to enhance their phagocytic activity. Target RBCs were double-labelled with fluorescent carboxyfluorescein-succinimidyl ester (CF-SE) and the DNA label ethidium bromide (EB). EB, a DNA label, allowed to discriminate p-RBCs that contain parasitic DNA from the np-RBCs devoid of DNA. FACS analysis of THP-1 cells fed with double-labelled RBCs showed that $p$ - and $n p-R B C s$ were phagocytosed in different proportions in relation to parasitaemia.

Conclusions: The assay allowed the analysis of phagocytosis rapidly and with low subjective error, and the differentiation between phagocytosed $\mathrm{p}$ - and $\mathrm{np}-\mathrm{RBC}$ in the same sample. The presented method may help to analyse the factors or conditions that modulate the share of $\mathrm{np}-\mathrm{RBC}$ removal in vitro and in vivo and lead to a better understanding of the pathogenesis of SMA.
\end{abstract}

Keywords: Malaria anaemia, Phagocytosis method, THP-1 cells, Phagocyte

\section{Background}

Severe malaria anaemia (SMA) is a frequent cause of mortality mainly in children and pregnant women [1-3]. The most important determinant of SMA appears to be the removal of non-parasitized red blood cells (np-RBCs) [4,5] most likely by intra- and extravascular phagocytosis [1].

\footnotetext{
* Correspondence: paolo.arese@unito.it

Department of Genetics, Biology and Biochemistry, University of Torino, Torino, Italy
}

The phagocytosis assay presented here utilizes the activated human phagocytic cell line THP-1 and the cytofluorimetric analysis of ingested p-RBCs and np-RBCs double-labelled with the fluorescent carboxyfluoresceinsuccinimidyl ester (CF-SE) and the DNA label ethidium bromide (EB) to determine phagocytosed np- and p-RBCs, respectively, in the same sample. The assay is very sensitive, can be performed rapidly with low numbers of $\mathrm{p}$ - and np-RBCs and overcomes the limitation of currently available methods that do not differentiate between phagocytosis of p-RBCs vs np-RBCs. 
In spite of the lack of quantitative data on the relative role of phagocytosis of $\mathrm{p}-v s \mathrm{np}-\mathrm{RBCs}$, the presented method may help to clarify the pathogenesis of SMA analysing the factors or conditions that induce and modulate the removal of np-RBC in vitro and in vivo in both falciparum and vivax malaria.

\section{Methods}

\section{Materials}

Unless otherwise indicated all reagents were from SigmaAldrich, St Louis, MO, USA. Penicillin-streptomycin was from Invitrogen, Carlsbad, CA, USA; TNF was from PeproTech Inc, Rocky Hill, NJ, USA; IFN $\gamma$ was from R\&D Systems, Minneapolis, MN, USA; bis[sulphosuccinimidyl] suberate, sodium salt $\left(\mathrm{BS}^{3}\right)$ was from Pierce Biotechnology Inc, Rockford, IL, USA; carboxyfluorescein diacetate succinimidyl ester (CFDA-SE), the non-fluorescent precursor of CF-SE, was from Fluka, Sigma-Aldrich, Milano, Italy; antiD IgG (Rhophylac ${ }^{\circledR}$ ) were from ZLB Behring SpA, Milano, Italy; Ficoll was from Biochrom AG, Berlin, Germany.

\section{Cultivation of Plasmodium falciparum and stage- dependent separation of parasites}

Plasmodium falciparum parasites (Palo Alto strain, Mycoplasma-free) were cultivated in RBCs from healthy donors at $2 \%$ haematocrit and synchronized as described [6]. Briefly, schizont-stage parasitized RBCs (parasitaemia >95\%) were mixed for invasion with washed RBCs and kept in growth medium (RPMI 1640 containing $25 \mathrm{mM}$ HEPES, $30 \mathrm{mM}$ glucose, $2 \mathrm{mM}$ glutamine, $0.025 \mathrm{mM}$ adenine, $24 \mathrm{mM} \mathrm{NaHCO}, 32 \mathrm{mg} / \mathrm{l}$ gentamicin and $10 \% \quad(\mathrm{vol} / \mathrm{vol}) \mathrm{A}^{+}$heat-inactivated human plasma) (time 0). After $20 \mathrm{hr}$ incubation in a humidified $\mathrm{CO}_{2}$ /air incubator, the ring-enriched fraction was separated on and collected from a discontinuous 40/80/90\% vol/vol Percoll gradient containing mannitol (6\% wt/vol). After further $20 \mathrm{hr}$ incubation under the same conditions, the trophozoite (troph)-enriched fraction was separated and collected as described before. Alternatively, ring- and troph-stages were separated by the same procedure from non-synchronized cultures of $\mathrm{p}$-RBCs and enriched to $60 \%$ and $90 \%$ parasitaemia, respectively. When needed, the culture was passed through 40\% (vol/ vol) Percoll containing 6\% mannitol (wt/vol) to remove residual bodies (RBs). Parasitaemia was assessed by light microscopy after Diff-Quik ${ }^{\circledR}$ Fix staining (Medion Diagnostics $\mathrm{GmbH}$, Düdingen, Switzerland). Control np-RBCs were incubated and treated in a similar way without schizont inoculation at time 0 .

\section{Fluorescent labelling of $\mathrm{p}$ - and $\mathrm{np}-\mathrm{RBCs}$ with CF-SE and ethidium bromide (EB)}

$15 \mu \mathrm{l}$ of packed $\mathrm{p}$ - or np-RBCs were suspended in $30 \mathrm{ml}$ of phosphate buffered saline-glucose (PBS-G; $150 \mathrm{mM}$
$\mathrm{NaCl}, 10 \mathrm{mM} \mathrm{Na} 2 \mathrm{HPO}_{4} / \mathrm{NaH}_{2} \mathrm{PO}_{4}, 2 \mathrm{mM}$ glucose, $\mathrm{pH}$ 7.4) containing $0.2 \mathrm{mM}$ carboxyfluorescein diacetate succinimidyl ester (CFDA-SE), the non fluorescent precursor of CF-SE, and incubated for $10 \mathrm{~min}$ at $37^{\circ} \mathrm{C}$. CFDA-SE is a non-fluorescent compound that passively diffuses into RBCs where it is de-acetylated to the high fluorescent CF-SE and stably retained within the RBC upon covalent binding to intracellular amino groups [7]. The labelling reaction was stopped by adding $15 \mathrm{ml}$ of heat-inactivated foetal bovine serum (FBS) for $5 \mathrm{~min}$. Thereafter the cells were washed three times with PBS$G$ and finally re-suspended in PBS-G for opsonization. EB labelling was performed by incubating $15 \mu \mathrm{l}$ of packed p- or np-RBCs in $135 \mu \mathrm{l}$ of PBS-G containing 1 $\mathrm{mg} / \mathrm{ml} \mathrm{EB}$ for $20 \mathrm{~min}$ at room temperature. The cells were then washed five times with PBS-G. In double labelling experiments $\mathrm{p}$ - or np-RBCs were incubated first with EB $1 \mathrm{mg} / \mathrm{ml}$ and then with CFDA-SE $0.2 \mathrm{mM}$ as described above. In order to minimize emission overlap of the two fluorochromes, a minor electronic compensation was applied.

\section{p- and np-RBC opsonization}

For phagocytosis, labelled p-and np-RBC, were opsonized with fresh homologous serum from healthy donors. RBCs were suspended at $33 \%$ haematocrit in fresh serum, diluted 1:1 (vol/vol) with PBS-G, incubated for $1 \mathrm{hr}$ at $37^{\circ} \mathrm{C}$ and subsequently washed twice and resuspended in PBS-G for the phagocytosis assay.

\section{Phagocytosis of positive control RBCs}

(a) Opsonization of np-RBCs with anti-D IgG: freshly drawn heparinized human blood was sedimented by centrifugation at $1,180 \mathrm{~g}$ for $5 \mathrm{~min}$ at room temperature and mononuclear cells, platelets and neutrophils removed by aspiration. Isolated RBCs were washed twice with PBS-G, labelled with CF-SE as indicated before, resuspended at 33\% haematocrit with anti-D IgG diluted 1:64 (vol/vol) with PBS-G, and incubated for $30 \mathrm{~min}$ at $37^{\circ} \mathrm{C}$. Negative control RBCs were incubated in PBS-G only. After opsonization, RBCs were washed twice and resuspended in PBS-G for the phagocytosis assay.

(b) Treatment of np-RBCs with zinc/BS ${ }^{3}$ : washed RBCs (see preceding section) were re-suspended at $10 \%$ haematocrit in HEPES-saline buffer $(10 \mathrm{mM}$ HEPES, 140 mM NaCl, 5 mM glucose, $\mathrm{pH}$ 7.4) containing $1 \mathrm{mM} \mathrm{ZnCl}$ and incubated for $10 \mathrm{~min}$ at room temperature. Thereafter the cross-linking agent $\mathrm{BS}^{3}$ (200 $\mathrm{mM}$ stock solution in dimethyl sulfoxide) was added to the RBC suspension to a final concentration of $1 \mathrm{mM}$. After $10 \mathrm{~min}$ incubation at room temperature, RBCs were washed 
twice in HEPES-saline buffer supplemented with 10 $\mathrm{mM}$ ethanolamine and then twice in HEPES-saline buffer supplemented with bovine serum-albumin (BSA, $1 \% \mathrm{wt} / \mathrm{vol}$ ). Washed zinc/BS 3 -treated RBCs and untreated control RBCs were then labelled with CF-SE, opsonized and resuspended in PBS-G for the phagocytosis assay.

\section{THP-1 cell cultivation and phagocytosis assay by flow cytometry}

Human monocytic THP-1 cells were grown in RPMI 1640 medium supplemented with $10 \%(\mathrm{vol} / \mathrm{vol})$ heat-inactivated foetal bovine serum (FBS), $2 \mathrm{mM} \mathrm{L}$-glutamine, $100 \mathrm{U} / \mathrm{ml}$ penicillin and $100 \mu \mathrm{g} / \mathrm{ml}$ streptomycin. Fresh medium was replaced twice a week. For general maintenance, the cells were seeded at $1 \times 10^{5}$ cells $/ \mathrm{ml}$. For phagocytosis, THP-1 cells were pre-activated by TNF $(250 \mathrm{U} / \mathrm{ml})$ and IFN $\gamma$ $(50 \mathrm{U} / \mathrm{ml})$ for $24 \mathrm{hr}$ in complete RPMI-1640 medium (RPMI 1640 medium supplemented with $10 \%$ (vol/vol) heat-inactivated FBS, $2 \mathrm{mM}$ L-glutamine, $100 \mathrm{U} / \mathrm{ml}$ penicillin and $100 \mu \mathrm{g} / \mathrm{ml}$ streptomycin) in a tissue culture polystyrene flask (Falcon, BD Biosciences, San Jose, CA, USA). Thereafter cells were harvested and washed three times with RPMI-1640 medium and finally re-suspended in phagocytosis medium (RPMI 1640 medium supplemented with $10 \%$ (vol $/ \mathrm{vol}$ ) FBS), to a concentration of $3 \times 10^{5}$ cells/300 $\mu \mathrm{l}$ medium. Pre-activated THP-1 cells were incubated for $150 \mathrm{~min}$ with RBCs $(1.5 \mu \mathrm{l}$ packed RBCs per $3 \times 10^{5}$ THP-1 cells) in $300 \mu$ lof phagocytosis medium, into round-bottomed $5 \mathrm{ml}$ polypropylene tubes (Falcon $\mathrm{BD}$ Biosciences) in a humidified $5 \% \mathrm{CO}_{2}$ incubator at $37^{\circ} \mathrm{C}$. As a negative control, THP- 1 cells were incubated in phagocytosis medium, with untreated RBCs kept at the same cultivation conditions. At the end of the phagocytosis period, the cell suspension was stratified on Ficoll $(1 \mathrm{ml})$ and subsequently centrifuged for $20 \mathrm{~min}$ at $914 \mathrm{~g}$ to separate THP-1 cells from not-phagocytosed RBCs. The THP-1 cells were harvested, washed once with RPMI 1640 medium, and resuspended in phagocytosis medium for analysis of phagocytosis by flow cytometry. The fluorescence of THP1 was acquired on a FACSCalibur flow cytometer (BD Biosciences, Sunnyvale, CA, USA) using the Cell Quest software and the files obtained were analysed with the WinMDI (The Scripps Research Institute, La Jolla, CA, USA) software. After gating the THP-1 cell population (physical parameters analysis) excluding contaminating not-phagocytosed RBCs and cell debris, phagocytosis was quantified by fluorescence intensity of the selected cell population. A total of at least 30,000 events were collected per gate for each sample. First, the percentage of phagocytically active THP-1 cells (phTHP-1) was assessed by counting the cells positive for CF-SE fluorescence. Secondly, the percentage of THP-1 cells that have phagocytosed p-RBC (EB positive cells) was quantified.
Thirdly, the number of RBCs phagocytosed per phTHP-1 was quantified by comparing the mean fluorescence intensity (MFI) value of a phTHP-1 cell to the MFI value of a labelled RBC.

\section{Statistical analysis}

Independent $\mathrm{t}$-tests were performed to compare percentages of phTHP-1 or numbers of RBCs phagocytosed per phTHP-1 in different $\mathrm{np}-$ and $\mathrm{p}-\mathrm{RBCs}$ populations. P-values $(<0.02,<0.002$ and $<0.001)$ were used to define statistical significance and shown when appropriate.

\section{Results}

\section{Optimization of phagocytosis assay}

\section{THP-1 pre-activation by TNF and IFN $y$ treatment}

The human monocytic THP-1 cells were selected as phagocytes because they express $\mathrm{Fc}$ and C3b receptors [8], both essential for phagocytosis of variously damaged or modified np-RBCs as well as p-RBCs [9]. In addition, THP-1 cells display constant characteristics over time, are easily cultivated and not expensive in their maintenance. The intrinsically low phagocytic activity of THP-1 cells was enhanced by pre-treatment with TNF and IFN $\gamma$, which increase phagocytic activity by upregulating Fc- and complement receptor expression without modifying phagocytic selectivity $[10,11]$. Pre-treatment with cytokines was optimized at $250 \mathrm{U}$ TNF and $50 \mathrm{U}$ IFN $\gamma$ per $\mathrm{ml}$ THP-1 cell suspension $\left(1 \times 10^{5}\right.$ cells $\left./ \mathrm{ml}\right)$ supplemented $24 \mathrm{hr}$ before starting the phagocytosis experiments. To minimize the phagocytosis-unrelated entrance into the phagocytes of non-activated CFDA-SE leaking out of the labelled RBCs [7] the phagocytosis time was shortened to 150 min obtaining a high phagocytosis rate as well as a negligible low non-phagocytosis-specific staining of the phagocytes. The RBC/THP-1 ratio was optimized at $50 / 1$, respectively (Additional file 1A-B), resulting in at least $90 \%$ of maximal phagocytosis and low fluorescence background.

\section{Fluorescent labelling of $n p$ - and $p-R B C s$ with CF-SE}

$\mathrm{Np}$ - and p-RBCs were incubated with CFDA-SE, a nonfluorescent compound that passively diffuses into RBCs where it is de-acetylated to the highly fluorescent CF-SE (carboxyfluorescein succinimidyl ester) and stably retained within the RBC upon covalent binding to intracellular amino groups [7]. Fluorescent RBC labelling reached a plateau after about $10 \mathrm{~min}$ and was dependent on CFDASE concentration and haematocrit of the RBCs suspension (Figure 1A). Labelling conditions were experimentally optimized at $0.2 \mathrm{mM}$ CFDA-SE, 5,000 RBC/ $\mu$ l corresponding to $0.05 \%$ haematocrit and $10 \mathrm{~min}$ incubation time. Excess of extracellular protein added after incubation stops further label entrance into RBCs. (see Methods for details). A typical labelling experiment of np-RBC, ring- 
A

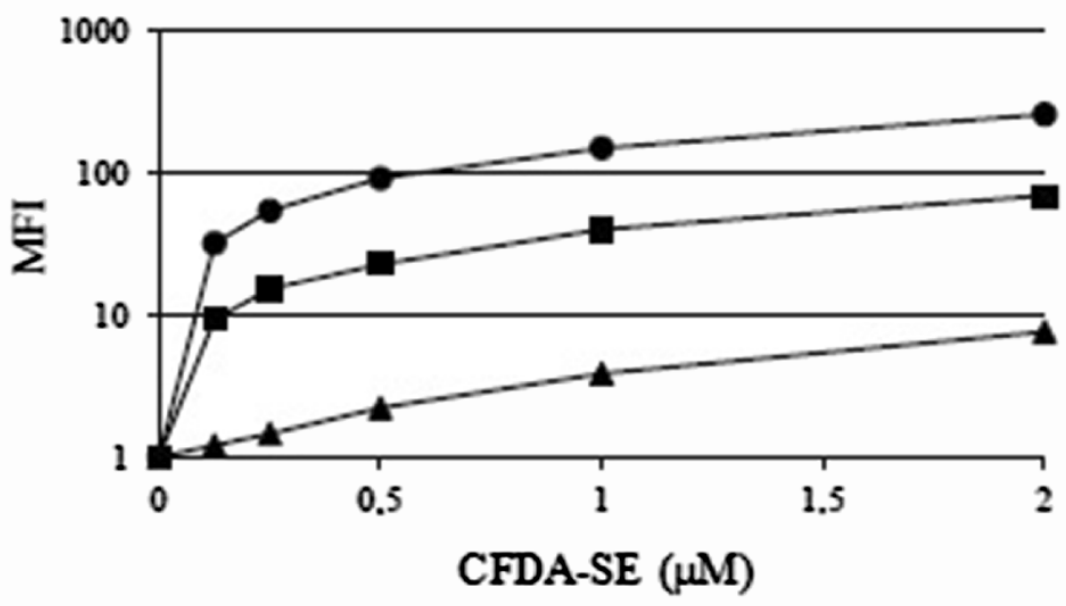

B

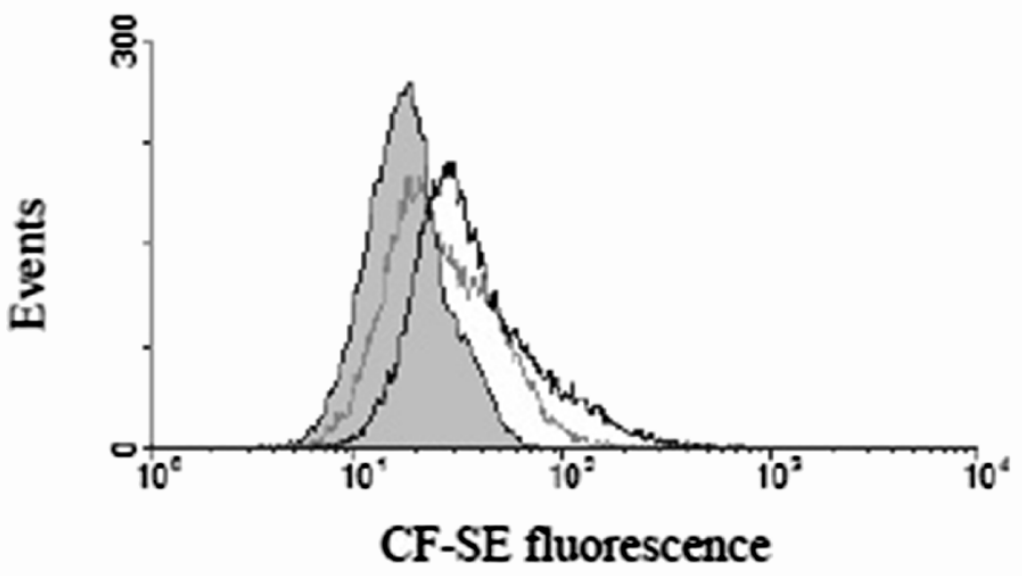

C

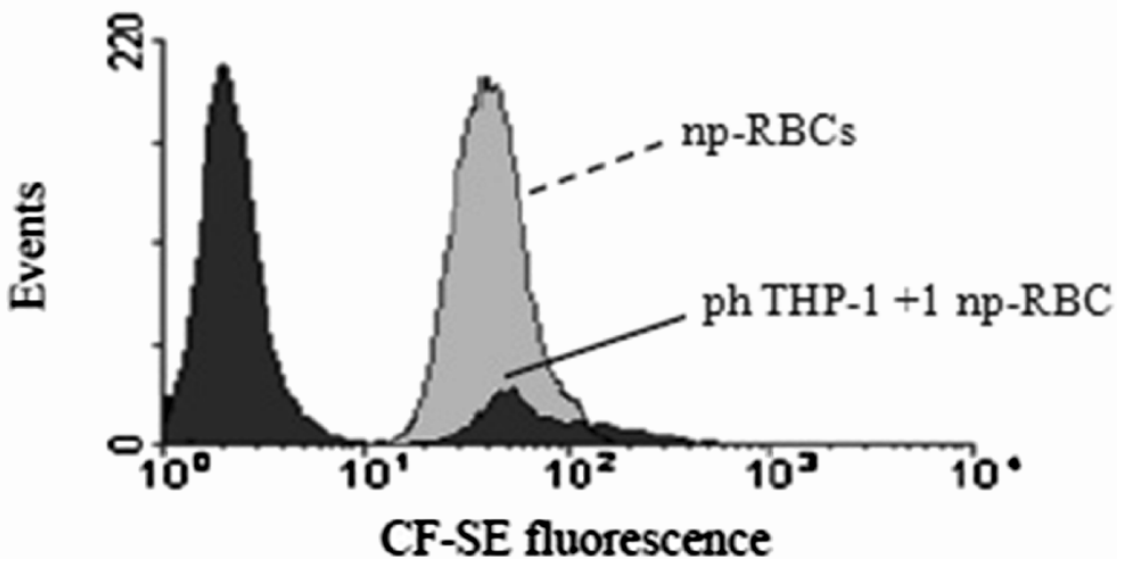

Figure 1 Fluorescent labelling of $\mathrm{np}$ - and p-RBCs with CF-SE. (A) Dependence of MFI on CFDA-SE concentration ( $\mu$ M final) and haematocrit in np-RBC. (black circle) 0.05\%, (black square) $0.5 \%$, (black triangle) $5 \%$ haematocrit, corresponding to $5 \times 10^{3}, 5 \times 10^{4}, 5 \times 10^{5} \mathrm{RBCs} / \mu \mathrm{l}$, respectively. (B) CF-SE fluorescence of np-RBCs (full grey histogram), ring-parasitized RBCs (light grey line), and troph-parasitized RBCs (dark grey line). np- and p-RBCs were incubated as indicated in (A). (C) Absence of fluorescence quenching in np-RBCs after phagocytosis. Fluorescence of CF-SE-labelled uningested np-RBCs (full grey histogram, MFI: $48.3 \pm 1.6, \mathrm{~N}=3$ ) and of phTHP-1 cells fed with limiting numbers of CF-SE-labelled np-RBCs (RBCs/ THP-1 ratio $=1$, full black histogram, MFI: $49.7 \pm 1.1, \mathrm{~N}=3$ ) were very similar. For details, see Methods. 
and troph-parasitized RBCs is shown in Figure $1 \mathrm{~B}$. Fluorescence intensity was substantially similar in np- and ring-parasitized RBCs, and distinctly higher in trophparasitized RBCs, possibly due to an increased uptake of the CFDA-SE or higher esterase activity in late stages of parasite development.

\section{Absence of fluorescence quenching after phagocytosis}

Quenching of the fluorescence output of CF-SE labelled RBCs after phagocytosis by THP-1 cells was checked. To this end THP-1 cells were fed with limiting numbers of anti-D IgG-opsonized np-RBCs (np-RBCs/THP-1 ratio $1: 1$, Figure $1 C$ ), assuming that on average each THP-1 cell had phagocytosed one np-RBC, as evidenced by the intensity peaks shown in Figure $1 \mathrm{C}$. The MFI of the THP-1 population containing one RBC was compared with the MFI of the labelled uningested np-RBCs. The fluorescence intensity of the two populations was similar: $49.7 \pm 1.1(\mathrm{~N}=3)$ and $48.3 \pm 1.6(\mathrm{~N}=3)$ for THP-1 that have ingested one RBC and uningested RBCs, respectively. Similar results were obtained with p-RBCs (not shown), allowing the conclusion that the fluorescence intensity was not modified within the phagocyte.

\section{Phagocytosis of positive control np-RBCs}

The method was first checked with positive control npRBCs. Two RBC treatments were utilized: first, np-RBCs were opsonized with anti-D IgG to stimulate IgGmediated recognition and phagocytosis via Fc-receptors by the phagocyte. Second, np-RBCs were treated with a combination of zinc, a band 3 clustering agent, and $\mathrm{BS}^{3}$, a cluster-stabilizing agent $\left(\mathrm{Zn} / \mathrm{BS}^{3}\right)$. Band 3 clusters elicit a limited activation of the complement pathway and induce a predominantly complement-mediated RBC recognition and removal [12]. Both treatments induce maximal removal of RBCs [13].

\section{Phagocytosis of anti-D lgG opsonized $n p-R B C s$}

Phagocytosis of anti-D IgG opsonized np-RBCs was significantly $(\mathrm{p}<0.001)$ increased compared to non-opsonized np-RBCs, both as percentage of phTHP-1 cells from $3.2 \pm$ $0.4 \%$ (non-opsonized, $\mathrm{N}=5$ ) to $56.9 \pm 3.4 \%$ (anti-D IgG opsonized cells, $\mathrm{N}=5$ ), (Figure $2 \mathrm{~A}$, first and second dot plot, respectively), and as the mean number of cells phagocytosed per phTHP-1 from $1.0 \pm 0.1$ (non-opsonized cells, $\mathrm{N}=5$ ) to $3.6 \pm 0.3$ (anti-D IgG opsonized cells, $\mathrm{N}=5$ ) (Figure 2B, first and second column, respectively).

\section{Phagocytosis of $\mathrm{Zn} / \mathrm{BS}^{3}$ treated $n p-R B C s$}

A second check with positive controls was performed with $\mathrm{np}-\mathrm{RBC}$ s treated with $\mathrm{Zn} / \mathrm{BS}^{3}$. Their phagocytosis was also significantly $(\mathrm{P}<0.002)$ increased compared to untreated np-RBCs, both as percentage of phTHP-1 cells from $3.2 \pm$ $0.4 \%$ (non-opsonized untreated cells, $\mathrm{N}=5$ to $25.1 \pm 3.2 \%$
$\left(\mathrm{Zn} / \mathrm{BS}^{3}\right.$ treated, serum-opsonized cells, $\mathrm{N}=5$ ) (Figure $2 \mathrm{~A}$, first and third dot plot, respectively), and as the mean number of cells phagocytosed per phTHP-1 from $1.0 \pm 0.1$ (non-opsonized untreated cells, $\mathrm{N}=5)$ to $2.1 \pm 0.3\left(\mathrm{Zn} / \mathrm{BS}^{3}\right.$ treated, serum opsonized cells, $\mathrm{N}=5$ ) (Figure 2B, first and third column, respectively, $\mathrm{P}<0.001)$. Control experiments demonstrated that serum opsonization is essential for phagocytosis induction, as $\mathrm{Zn} / \mathrm{BS}^{3}$ treated, non-opsonized RBCs were not or poorly phagocytosed (Figure $2 \mathrm{~A}$, fourth plot, Figure 2B, fourth column).

\section{Phagocytosis of stage-separated $p$ - and np-RBCs single-labelled with CF-SE}

Phagocytosis experiments were performed using synchronized ring- and troph-enriched fractions after Percoll gradient separation $20 \mathrm{hr}$ and $40 \mathrm{hr}$ after reinvasion, resulting in approximately $60 \%$ and $>90 \%$ rings and trophozoites respectively. $\mathrm{p}$ - and $\mathrm{np}-\mathrm{RBCs}$ from the same donor were stained with CF-SE, opsonized with human homologous serum (np-RBCs) and fed to THP-1 cells as detailed in Materials. Phagocytosis of p-RBCs increased stage-dependently either as percentage of phTHP-1 cells or as mean number of p-RBCs ingested per phTHP-1. phTHP-1 cells increased from $6.8 \pm 1.7 \%(\mathrm{np}-\mathrm{RBCs}, \mathrm{N}=5)$ to $20.8 \pm$ $2.2 \%$ (ring, $\mathrm{N}=5$ ) and $50.6 \pm 4.4 \%$ (troph, $\mathrm{N}=5$ ) (Figure $3 \mathrm{~A}$, all $\mathrm{P}<0.001$ ), while $\mathrm{p}-\mathrm{RBC}$ ser THP-1 cell increased from $1.2 \pm 0.3(\mathrm{np}-\mathrm{RBCs}, \mathrm{N}=5)$ to $1.8 \pm 0.2$ (ring, $\mathrm{N}=5$ ), and to $3.5 \pm 0.4$ (troph, $\mathrm{N}=5$ ) (Figure $3 \mathrm{~B}$, troph $v s$ ring and $\mathrm{np}-\mathrm{RBCs}, \mathrm{P}<0.001$, ring vs np-RBCs, $\mathrm{P}<0.02)$.

\section{Exclusion of artifacts}

To exclude the presence of intact RBCs adherent to the THP-1 surface, ice-cold distilled water was added briefly to the cells after the phagocytosis period and immediately before FACS analysis. Physical parameters, fluorescence and phagocytosis data were not significantly changed by the treatment excluding the presence of intact RBCs adherent to the THP-1 surface (Figure 4A), indicating that the Ficoll passage after phagocytosis is sufficient to efficiently remove RBCs. Former observations with human monocytes fed with CF-SE labelled RBCs [14] indicate that no fluorescence was associated to membranes. Secondly, quenching activity by residual bodies (RBs), free haemozoin expelled from the mature schizont during merozoites release in the cultures was examined. RBs had no influence on phagocytosis quantification as indicated by invariant results shown in Figure 4B. Therefore, presented assay seems to be suitable for both RBs-depleted and RBs-containing cultures and for ex vivo analysis without further parasite separation. 


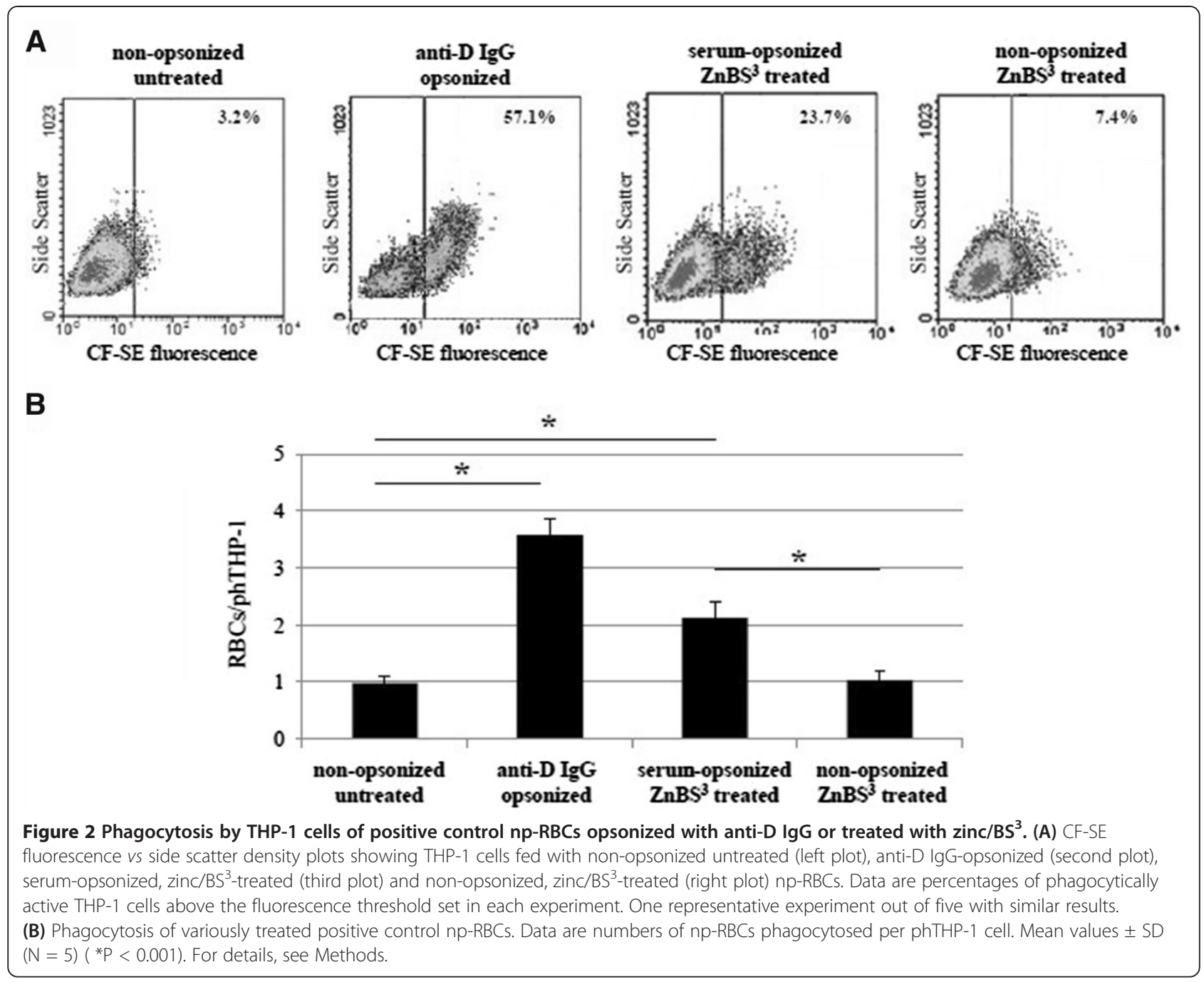

Phagocytosis of $p$ - and np-RBCs double-labelled with CF-SE and $E B$

In double-labelling experiments, $\mathrm{np}$ - and $\mathrm{p}-\mathrm{RBCs}$ were labelled with ethidium bromide (EB), before CF-SE labelling. EB, a DNA intercalating agent, develops red fluorescence after binding to DNA. Therefore, EB was used to distinguish DNA-free np-RBCs from p-RBCs, which contain parasite DNA. Labelling conditions were optimized at $1 \mathrm{mg} / \mathrm{ml} \mathrm{EB}, 10 \%$ haematocrit $\left(1 \times 10^{6} \mathrm{RBCs} / \mu \mathrm{l}\right)$ and $20 \mathrm{~min}$ incubation time. Figure $5 \mathrm{~A}$ shows the $\mathrm{EB}$ fluorescence of a synchronized trophozoite cultures with $44 \%$ parasitaemia. The difference between np-RBCs and troph-pRBCs reflects absence and presence of DNA, respectively.

The aim of double staining with CF-SE and EB was to discriminate between phagocytosis of $\mathrm{p}$ - and np-RBCs in cultures containing both cell types, as only p-RBCs were stained by EB. FACS analysis of a THP-1 population fed with double-labelled RBCs clearly shows that $\mathrm{p}$ - and
$\mathrm{np}$-RBCs were phagocytosed in different proportions. Figure 5B-D shows the phagocytosis of two synchronized cultures at trophozoite stage and approximately $8 \%$ and $44 \%$ parasitaemia. Opsonization with homologous serum increased phTHP-1 from 9.25\% and 20.9\% (non-opsonized RBCs, $8 \%$ and $44 \%$ parasitaemia, respectively) to $24.14 \%$ and $55.51 \%$ (serum-opsonized RBCs, $8 \%$ and $44 \%$ parasitaemia, respectively). EB staining allowed to ascertain the share of $\mathrm{p}$ - and np-RBCs ingested at different parasitaemia values. As expected, at $44 \%$ parasitaemia the share of ingested p-RBC increased remarkably in both opsonized and non-opsonized samples. As shown in Figure 5C, 1.1\% (8\% parasitaemia) and 5.6\% (44\% parasitaemia) of phTHP1 had phagocytosed at least one non-opsonized p-RBCs, while $2.85 \%$ (8\% parasitaemia) and $22.68 \%$ (44\% parasitaemia) of phTHP-1 had phagocytosed at least one serumopsonized p-RBC. The mean number of RBCs (sum of p- plus np-RBCs) phagocytosed per phTHP-1 was also determined at low and high parasitaemia (Figure 5D). 


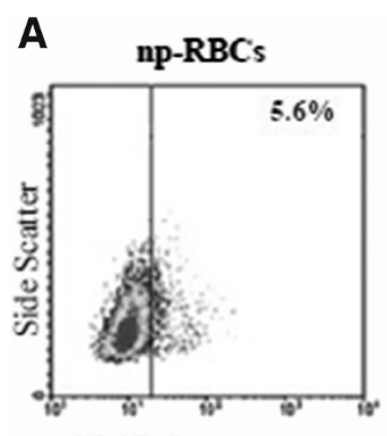

CF-SE fluorescence
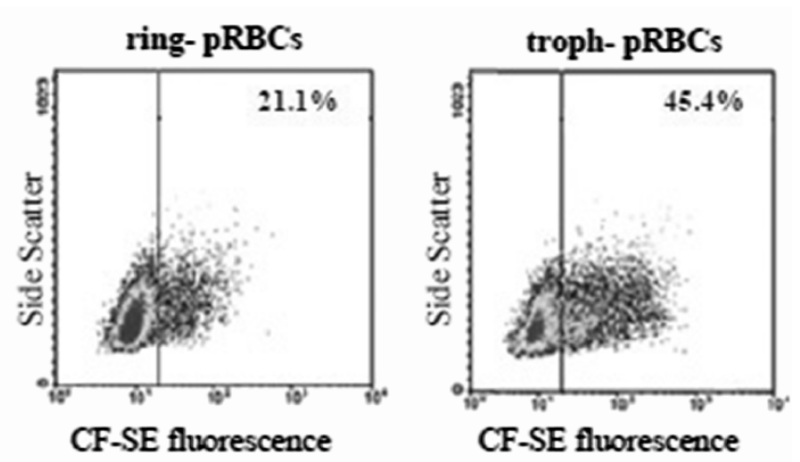

$\mathbf{B}$

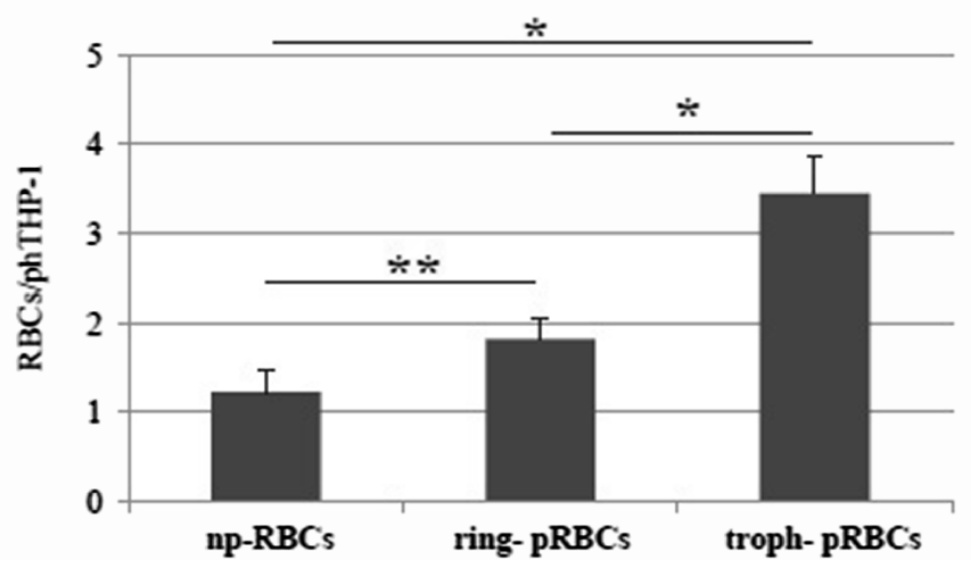

Figure 3 Phagocytosis by THP-1 cells of stage-separated np- and p-RBCs single-labelled with CF-SE. (A) Fluorescence vs side scatter density plots showing THP-1 cells fed with of serum-opsonized np-RBCs (left panel), ring-pRBCs (middle panel) and troph-pRBCs (right panel). Data are percentages of phagocytically active THP-1 cells above the fluorescence threshold set in each experiment. One representative experiment out of five with similar results. (B) Phagocytosis by THP-1 cells of CFDA-SE-labelled np-, ring- and troph-pRBCs. Data are numbers of RBCs ingested per THP-1 cell. Mean values $\pm S D(N=5)$. Phagocytosis of troph-pRBCs vs np-RBCs and vs ring-pRBCs, and phagocytosis of ring- vs np-RBCs was significantly higher with $\mathrm{P}<0.001^{*}$, and $\mathrm{P}<0.02^{* *}$, respectively. For details, see Methods.

To make sure that EB did not interfere with the phagocytosis quantification by CF-SE fluorescence, experiments were performed with np-RBCs stained with CF-SE only, or with both EB and CF-SE. Results indicated no significant difference between the two conditions (not shown). In cultures containing both $\mathrm{p}$ - and np-RBCs, quantification of phagocytosis using CF-SE labelling only did not discriminate between phagocytosed p- or np-RBCs. By contrast, additional DNA labelling by EB can discriminate between $\mathrm{p}$ - and np-cells and allowed to simultaneously quantify both the total number of RBCs ingested per phTHP-1 cell as well as the ratio between ingested p- and np-RBCs.

\section{Discussion}

This study describes a rapid, sensitive, reproducible and accurate assay to quantify phagocytosis of $\mathrm{p}$ - and np-RBCs in the same sample using CF-SE- and EB-labelled target cells and the human phagocytic monocyte cell line THP-1. Phagocytes were previously activated with TNF and IFN $\gamma$ to upregulate Fc- and complement receptors and enhance phagocytic efficiency without loss of specificity $[10,11]$. Target RBCs were double-labelled with CF-SE and EB. CF-SE is the fluorescent derivative of CFDA-SE, a nonfluorescent lipophilic molecule that passively diffuses into the cell where it is activated by esterase cleavage of its acetyl groups to the brightly fluorescent derivative CF-SE. The latter, a non-toxic molecule, is stably retained in the cell-forming covalent conjugates to free amino groups, emits stable and homogeneous fluorescence and does not interfere with RBC functionality $[7,15]$. CF-SE does not localize in cell membranes and does not elicit membrane alterations that may induce phagocytic recognition of the labelled cells. EB, a widely used DNA fluorescent label, allows to discriminate $\mathrm{p}-\mathrm{RBCs}$ that contain parasitic DNA from the np-RBCs totally devoid of DNA.

The phagocytic efficiency of the THP-1 cells was checked with np-RBCs modified by two treatments known to maximally enhance phagocytosis: opsonization with anti-D IgG to stimulate IgG-mediated phagocytosis, 

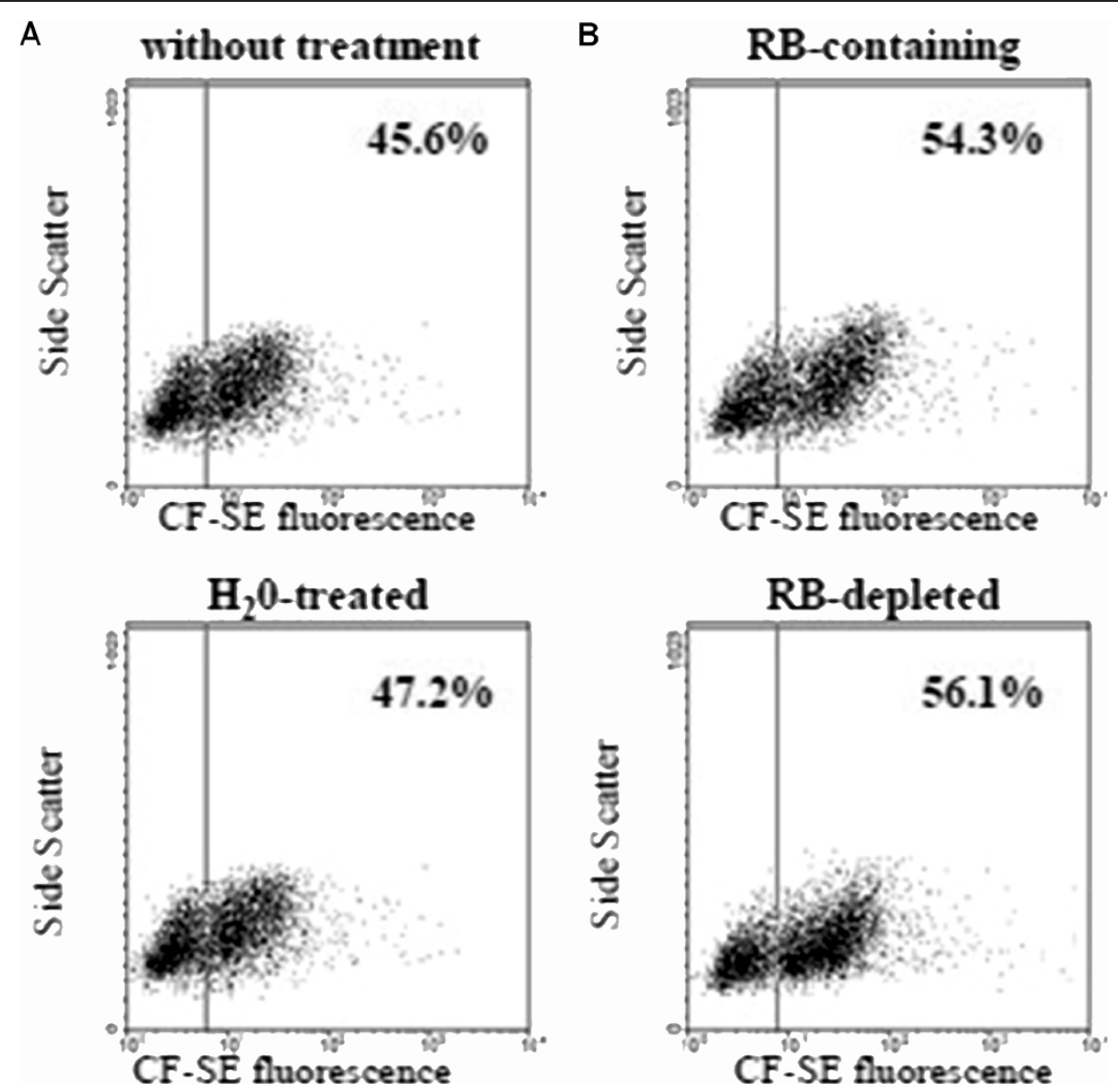

Figure 4 Exclusion of artifacts caused by adherence of RBCs and residual bodies to THP-1 cells. (A) Fluorescence of THP-1 cells fed with np-RBC-fed treated or not with water to lyse adherent intact RBCs. (B) Fluorescence of THP-1 cells fed with p-RBC in presence or absence of residual bodies (RBs). (A, B) CF-SE fluorescence vs side scatter dot plots of THP-1 cells fed with treated or untreated RBCs are very similar excluding artefactual adherence of RBCs or RBs to THP-1 cells. One representative experiment out of four (A) or three (B) with similar results. Data are percentages of phagocytically active THP-1 cells counted above the fluorescence threshold set in each experiment (for details, see Methods).

and $\mathrm{RBCs}$ treatment with zinc-BS ${ }^{3}$, that generates stable band-3 clusters and induce a predominantly IgG/complement-mediated phagocytosis [9]. Phagocytosis by activated THP-1 cells was lower compared to phagocytosis by adherent human monocytes $[9,16]$. However, THP-1 cells displayed the same specificity and relative phagocytic activity with respect to p-RBCs as human monocytes, while presenting a number of advantages, such as independence from availability of human buffy-coats, constant performance, low cost and easy maintenance.

Removal of large numbers of np-RBCs accompanies the rupture of $\mathrm{p}$-RBCs at schizogony, as shown by the simultaneous presence of $\mathrm{p}$-and $\mathrm{np}-\mathrm{RBCs}$ in the same phagocytic cell in peripheral blood and organ phagocytes [17-19] and by studies on the clearance kinetics of $\mathrm{p}$ - and np-RBCs $[20,21]$. It is generally maintained that $n p-R B C s$ are cleared in approximately 10 -fold excess compared to elimination of $\mathrm{p}-\mathrm{RBC}$ at schizogony. However, this estimate is based on a single study [5], performed by modelling 12 neurosyphilitic patients who underwent malaria therapy. Those authors observed a substantial destruction of np-RBCs occurring during two cycles of parasitaemia with peak values at 20,000-40,000 parasites per $\mu \mathrm{l}$ and estimated that an average of 8.5 $n p-R B C s$ were destroyed per rupturing schizont. An analogous study [22] has described an even larger excess removal of np-RBCs in vivax malaria anaemia, a frequent and severe complication of vivax infection occurring by largely undisclosed mechanism [23,24].

Apart from RBC lysis at schizogony, it is assumed that phagocytosis is the predominant mechanism of removal of np- and p-RBC. This assertion is based on a balance study in acute SMA where a quantitative comparison was performed between the loss of blood haemoglobin $(\mathrm{Hb})$ and increase of $\mathrm{Hb}$ in plasma and urine. Increase in plasma $\mathrm{Hb}$ was less than $1 \%$ and excretion of $\mathrm{Hb}$ in urine was less than $0.5 \%$ of total $\mathrm{Hb}$ loss [25]. This data tends to exclude complement lysis [26] and is in line 


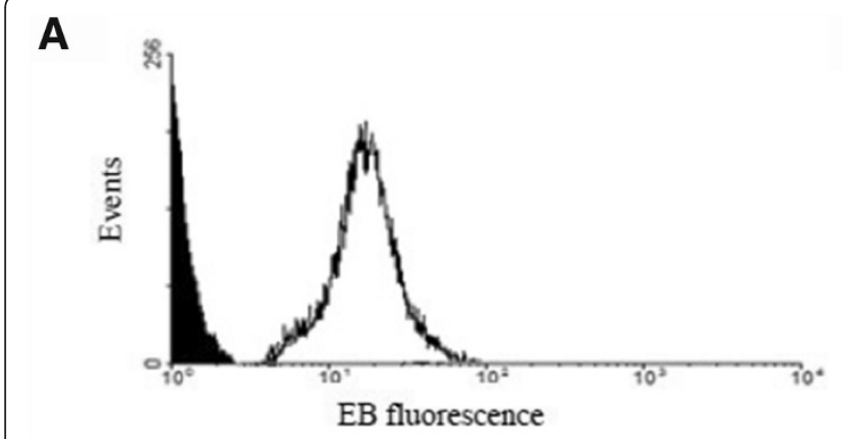

B
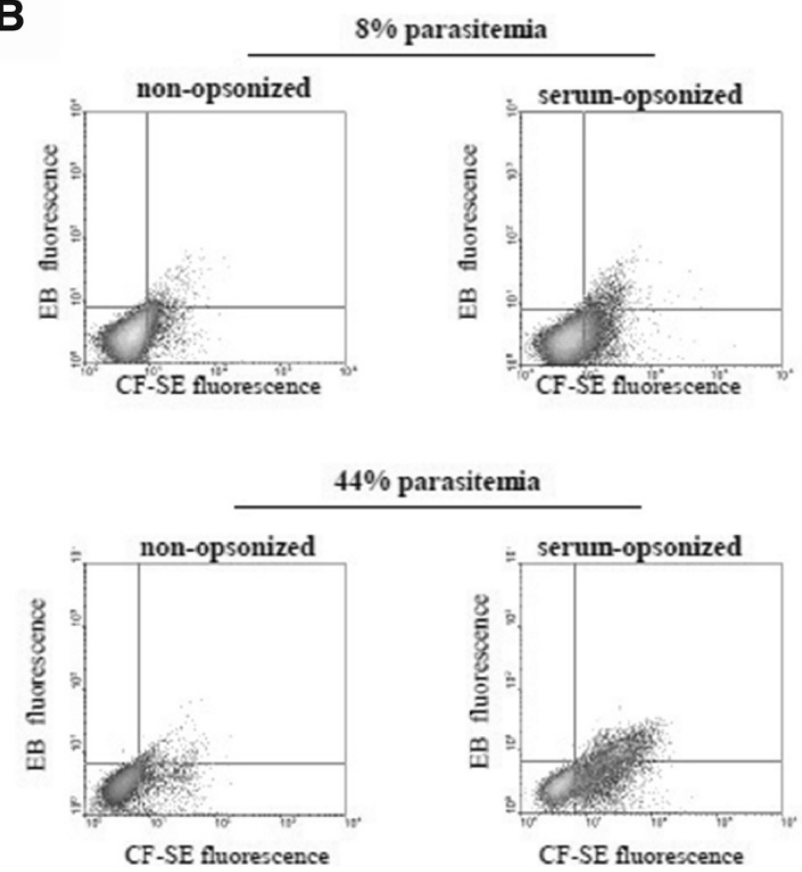

$44 \%$ parasitemia

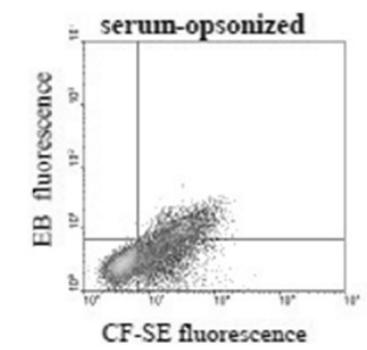

\section{C}

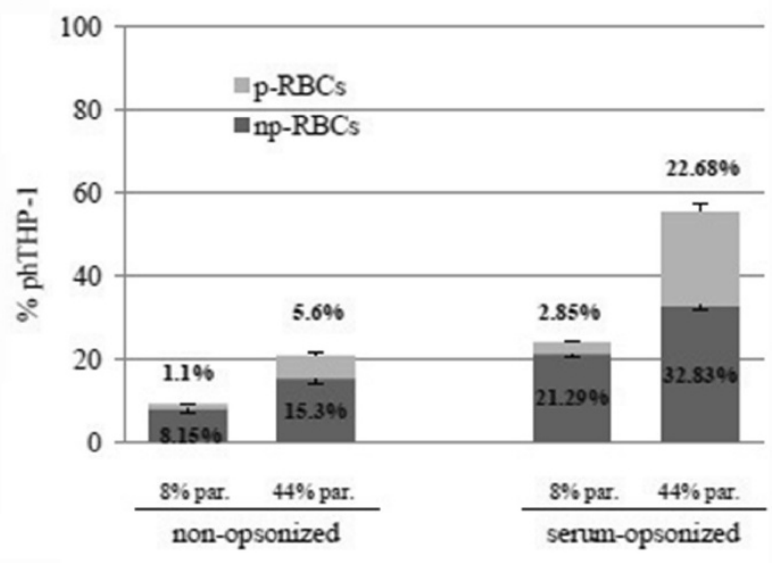

D

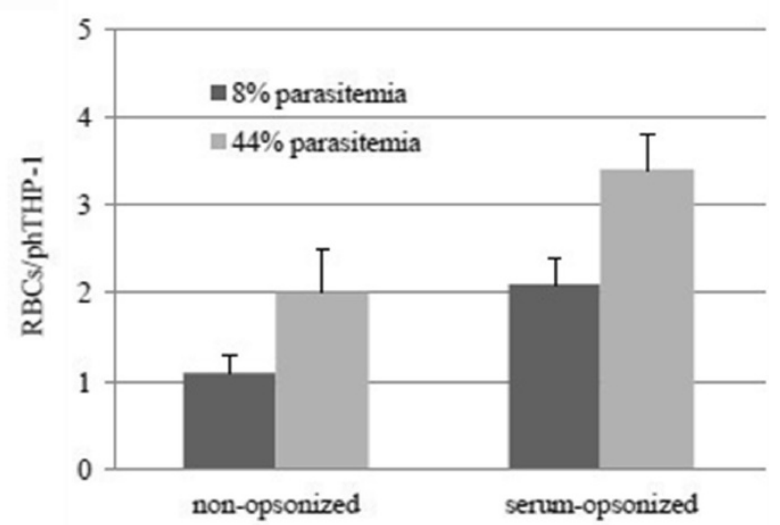

Figure 5 Phagocytosis of troph-p-RBCs and np-RBCs double-labelled with EB and CF-SE. (A) EB fluorescence of a synchronized trophozoite culture with 44\% parasitaemia: troph-p-RBCs, black line, np-RBCs, full histogram. (B) CF-SE fluorescence vs EB fluorescence density plots of THP-1 cells fed with non-opsonized or serum-opsonized synchronized trophozoite cultures with $8 \%$ and $44 \%$ parasitaemia. In both upper (8\% parasitaemia) and lower (44\% parasitaemia) plots, upper and lower right quadrants show phagocytically active THP-1 cells fed with troph-p-RBCs or np-RBCs, respectively. Cells positive for CF-SE and EB were counted above the fluorescence threshold set in each experiment. One representative experiment out of six with similar results. (C) Phagocytosis of serum-opsonized and non-opsonized troph-p- and np-RBCs by THP-1 cells in synchronized trophozoite cultures with $8 \%$ and $44 \%$ parasitaemia. Numbers on bars are percentages of phagocytically active (ph)THP-1 cells. For each phTHP-1 value the share of $\mathrm{p}$ - and np-RBCs in the sample is indicated. Bar data are mean values of phTHP-1 cells \pm SD (N=6). (D) Total phagocytosis of serum-opsonized and non-opsonized p- plus np-RBCs by THP-1 cells in synchronized trophozoite cultures with $8 \%$ and 44\% parasitaemia. Data are numbers of $p$ - plus np-RBCs phagocytosed per phTHP-1 cell. Bar data are mean values $\pm S D(N=6)$. For details, see Methods.

with the common paradigm of phagocytic removal of senescent, variously damaged RBCs and falciparum p$\mathrm{RBCs}$ as the consequence of membrane damage and opsonization through enhanced binding of complement factor C3b and (anti-band 3)-IgG [9,27,28].

Most available cytofluorimetric methods (for example: $[14,29])$ do not allow to discriminate the relative contribution of np- vs p-RBC removal. One exception, however, is the method by Tippett et al [30] designed to assess phagocytosis by patient monocytes or by THP- 1 cells of $\mathrm{p}$ - and
np-RBCs simultaneously labelled by EB and fluorescein isothiocyanate (FITC). Comparison of our results with Tippett et al' is difficult because they used unstimulated THP-1 cells with low phagocytic activity and FITC, a membrane-standing molecule known to interfere with RBC membrane transport systems and surface carbohydrates [31,32]. In few cases though, a comparison was possible. For example, the percentage of phagocytically active cells was between nil and 10\% in unstimulated [30], and $45 \%$ in pre-stimulated [this study] THP- 1 cells when both 
TPH-1 cells were challenged with serum-opsonized pRBCs. Higher phagocytic activity was also noted when RBC suspensions with 3-8\% p-RBCs were challenged by unstimulated [30] or pre-stimulated [this study] THP-1 cells. Phagocytically active cells were approx. $2 \%$ in unstimulated and approx. $24 \%$ pre-stimulated THP-1 cells.

In conclusion, due to the lack of reliable quantitative data on the relative role of phagocytosis of $\mathrm{p}-v s \mathrm{np}-\mathrm{RBCs}$, the method presented here may help to fill gaps particularly in the pathogenesis of SMA. For example, it will be useful to analyse the factors or conditions that modulate the share of np-RBC removal in vitro and in vivo. It appears that np-RBC removal may vary within broad limits as documented in human SMA and in humanized murine models and thus be an important determinant of SMA [33,34]. The causes for this variability are unknown. Possible factors are bystander modifications that induce RBC membrane modifications, transfer of toxic parasiteproduced molecules in rosettes [35], malaria- or agerelated variations in phagocytosis-enhancing complement factors [26,36] and surface IgG [37], and upregulation of phagocytic activity of host phagocytes [20]. Phagocytosis studies ex vivo may also help to clarify the mechanism of malaria protection against severe anaemia observed in correlative studies in alpha-thalassaemia patients characterized by minimal alterations of RBC morphology, functionality and $\mathrm{RBC}$ lifespan in the np-status.

\section{Additional file}

\section{Additional file 1: Optimization of phagocytosis assay. (A)}

Dependence of phagocytosis on phagocytosis time. Cell ratio: 50 np-RBCs per THP-1 cell. (B) Dependence of phagocytosis on np-RBCs/ THP-1 cell ratio. Phagocytosis time: 150 min. CF-SE-labelled, lgG anti-D-opsonized np-RBCs were exposed at indicated cell ratios to pre-activated THP-1 cells for various phagocytosis periods. Phagocytosis is expressed as percentage of phagocytically-active THP-1 (phTHP-1). Mean values $\pm S D(N=4)$. For details, see Methods.

\section{Competing interests}

The authors declare that they have no competing interests.

\section{Authors' contributions}

VG, PA, OAS and ES designed experiments; VG and OA performed experiments; VG and PA drafted the manuscript; all authors discussed experiments and results and commented on the manuscript. All authors read and approved the final manuscript.

\section{Acknowledgements}

This study was supported by grants provided to VG and OS by University of Torino, to ES by the Italian Ministry of Education, University and Research (PRIN Project 2008) and to PA by the European Virtual Institute dedicated to Malaria Research (EVIMalaR), Grant Agreement FP7 242095.

Received: 10 July 2012 Accepted: 30 October 2012

Published: 21 December 2012

\section{References}

1. Ekvall H: Malaria and anemia. Curr Opin Hematol 2003, 10:108-114.

2. Casals-Pascual C, Roberts DJ: Severe malarial anaemia. Curr Mol Med 2006, 6:155-168.
3. Murphy SC, Breman JG: Gaps in the childhood malaria burden in Africa: cerebral malaria, neurological sequelae, anemia, respiratory distress, hypoglycemia, and complications of pregnancy. AmJTrop Med Hyg 2001, 64:57-67.

4. Zuckerman A: Autoimmunization and other types of indirect damage to host cells as factors in certain protozoan diseases. Exp Parasitol 1964, 15:138-183.

5. Jakeman G, Saul A, Hogarth W, Collins W: Anaemia of acute malaria infections in non-immune patients primarily results from destruction of uninfected erythrocytes. Parasitology 1999, 119(Pt 2):127-133.

6. Skorokhod O, Schwarzer E, Ceretto M, Arese P: Malarial pigment haemozoin, IFN-gamma, TNF-alpha, IL-1 beta and LPS do not stimulate expression of inducible nitric oxide synthase and production of nitric oxide in immuno-purified human monocytes. Malar J 2007, 6:73.

7. Wang X, Duan X, Liu L, Fang Y, Tan Y: Carboxyfluorescein diacetate succinimidyl ester fluorescent dye for cell labeling. Acta Biochim Biophys Sin (Shanghai) 2005, 37:379-385.

8. Tsuchiya S, Yamabe M, Yamaguchi Y, Kobayashi Y, Konno T, Tada K: Establishment and characterization of a human acute monocytic leukemia cell line (THP-1). Int I Cancer 1980, 26:171-176.

9. Turrini F, Ginsburg H, Bussolino F, Pescarmona G, Serra M, Arese P: Phagocytosis of Plasmodium falciparum-infected human red blood cells by human monocytes: involvement of immune and nonimmune determinants and dependence on parasite developmental stage. Blood 1992, 80:801-808.

10. Drevets D, Leenen P, Campbell P: Complement receptor type 3 mediates phagocytosis and killing of Listeria monocytogenes by a TNF-alpha- and IFN-gamma-stimulated macrophage precursor hybrid. Cell Immunol 1996, 169:1-6.

11. Kitagawa S, Yuo A, Yagisawa M, Azuma E, Yoshida M, Furukawa Y, Takahashi M, Masuyama J, Takaku F: Activation of human monocyte functions by tumor necrosis factor: rapid priming for enhanced release of superoxide and erythrophagocytosis, but no direct triggering of superoxide release. Exp Hematol 1996, 24:559-567.

12. Turrini F, Arese P, Yuan J, Low P: Clustering of integral membrane proteins of the human erythrocyte membrane stimulates autologous IgG binding, complement deposition, and phagocytosis. J Biol Chem 1991, 266:23611-23617.

13. Arese $P$, Turrini F, Schwarzer E: Band 3/complement-mediated recognition and removal of normally senescent and pathological human erythrocytes. Cell Physiol Biochem 2005, 16:133-146.

14. Fendel R, Mordmüller B, Kreidenweiss A, Rudat A, Steur C, Ambrosch C, Kirstein M, Berdel W, Kremsner P, Brandts C: New method to quantify erythrophagocytosis by autologous monocytes. Cytometry A 2007, 71:258-264.

15. Lyons AB: Analysing cell division in vivo and in vitro using flow cytometric measurement of CFSE dye dilution. I Immunol Methods 2000, 243:147-154.

16. Schwarzer $E$, Turrini F, Arese P: A luminescence method for the quantitative determination of phagocytosis of erythrocytes, of malaria-parasitized erythrocytes and of malarial pigment. Br J Haematol 1994, 88:740-745.

17. Taliaferro $\mathrm{W}$, Mulligan $\mathrm{H}$ : The histopathology of malaria with special reference to the function and origin of macrophages in defense. Indian Med Res Mem 1937, 29:1-125.

18. Facer CA, Brown J: Monocyte erythrophagocytosis in falciparum malaria. Lancet 1981, 1:897-898.

19. Abdalla SH: Peripheral blood and bone marrow leucocytes in Gambian children with malaria: numerical changes and evaluation of phagocytosis. Ann Trop Paediatr 1988, 8:250-258.

20. Looareesuwan S, Merry AH, Phillips RE, Pleehachinda R, Wattanagoon Y, Ho M, Charoenlarp P, Warrell DA, Weatherall DJ: Reduced erythrocyte survival following clearance of malarial parasitaemia in Thai patients. $\mathrm{Br}\rfloor$ Haematol 1987, 67:473-478.

21. Looareesuwan S, Davis TM, Pukrittayakamee S, Supanaranond W, Desakorn V, Silamut K, Krishna S, Boonamrung S, White NJ: Erythrocyte survival in severe falciparum malaria. Acta Trop 1991, 48:263-270.

22. Collins WE, Jeffery GM, Roberts JM: A retrospective examination of anemia during infection of humans with Plasmodium vivax. AmJTrop Med Hyg 2003, 68:410-412.

23. Douglas NM, Anstey NM, Buffet PA, Poespoprodjo JR, Yeo TW, White NJ, Price RN: The anaemia of Plasmodium vivax malaria. Malar J 2012, 11:135. 
24. Mueller I, Galinski MR, Baird JK, Carlton JM, Kochar DK, Alonso PL, del Portillo HA: Key gaps in the knowledge of Plasmodium vivax, a neglected human malaria parasite. Lancet Infect Dis 2009, 9:555-566.

25. Ekvall H, Arese P, Turrini F, Ayi K, Mannu F, Premji Z, Bjorkman A: Acute haemolysis in childhood falciparum malaria. Trans R Soc Trop Med Hyg 2001, 95:611-617.

26. Odhiambo CO, Otieno W, Adhiambo C, Odera MM, Stoute JA: Increased deposition of C3b on red cells with low CR1 and CD55 in a malaria-endemic region of western Kenya: implications for the development of severe anemia. BMC Med 2008, 6:23.

27. Lutz HU: Innate immune and non-immune mediators of erythrocyte clearance. Cell Mol Biol (Noisy-le-Grand) 2004, 50:107-116.

28. Giribaldi G, Ulliers D, Mannu F, Arese P, Turrini F: Growth of Plasmodium falciparum induces stage-dependent haemichrome formation, oxidative aggregation of band 3, membrane deposition of complement and antibodies, and phagocytosis of parasitized erythrocytes. $\mathrm{Br} J$ Haematol 2001, 113:492-499.

29. Bratosin D, Mazurier J, Slomianny C, Aminoff D, Montreuil J: Molecular mechanisms of erythrophagocytosis: flow cytometric quantitation of in vitro erythrocyte phagocytosis by macrophages. Cytometry 1997, 30:269-274

30. Tippett E, Fernandes L, Rogerson S, Jaworowski A: A novel flow cytometric phagocytosis assay of malaria-infected erythrocytes. J Immunol Methods 2007, 325:42-50

31. Muallem S, Karlish SJ: Catalytic and regulatory ATP-binding sites of the red cell $\mathrm{Ca} 2+$ pump studied by irreversible modification with fluorescein isothiocyanate. J Biol Chem 1983, 258:169-175.

32. Järnefelt J, Laurent T, Rigler R: Diffusion of fluorescein-labelled molecules in suspensions of erythrocyte ghosts. FEBS Lett 1988, 242:129-133.

33. Price RN, Simpson JA, Nosten F, Luxemburger C, Hkirjaroen L, ter Kuile F, Chongsuphajaisiddhi T, White NJ: Factors contributing to anemia after uncomplicated falciparum malaria. AmJTrop Med Hyg 2001, 65:614-622.

34. Evans K, Hansen DS, van Rooijen N, Buckingham LA, Schofield L: Severe malarial anemia of low parasite burden in rodent models results from accelerated clearance of uninfected erythrocytes. Blood 2006, 107:1192-1199.

35. Uyoga S, Skorokhod OA, Opiyo M, Orori EN, Williams TN, Arese P, Schwarzer E: Transfer of 4-hydroxynonenal from parasitized to non-parasitized erythrocytes in rosettes. Proposed role in severe malaria anemia. $\mathrm{Br} \mathrm{J}$ Haematol 2012, 157:116-124

36. Goka BQ, Kwarko H, Kurtzhals JA, Gyan B, Ofori-Adjei E, Ohene SA, Hviid L, Akanmori BD, Neequaye J: Complement binding to erythrocytes is associated with macrophage activation and reduced haemoglobin in Plasmodium falciparum malaria. Trans R Soc Trop Med Hyg 2001, 95:545-549

37. Waitumbi JN, Opollo MO, Muga RO, Misore AO, Stoute JA: Red cell surface changes and erythrophagocytosis in children with severe Plasmodium falciparum anemia. Blood 2000, 95:1481-1486.

doi:10.1186/1475-2875-11-428

Cite this article as: Gallo et al: Simultaneous determination of phagocytosis of Plasmodium falciparum-parasitized and non-parasitized red blood cells by flow cytometry. Malaria Journal 2012 11:428.

\section{Submit your next manuscript to BioMed Central and take full advantage of:}

- Convenient online submission

- Thorough peer review

- No space constraints or color figure charges

- Immediate publication on acceptance

- Inclusion in PubMed, CAS, Scopus and Google Scholar

- Research which is freely available for redistribution

Submit your manuscript at www.biomedcentral.com/submit
C Biomed Central 